\title{
Tecnura
}

\section{Control y administración de datos en una mina subterránea de oro y plata}

\section{Control and administration of data in a gold and silver underground mine}

\author{
David Alfredo Narváez ${ }^{1(0)}$, Beatríz Lilian Pérez ${ }^{2(\infty)}$, Andrea Alejandra Giubergia ${ }^{3(\mathbb{1}}$, \\ Graciela Verónica Gil-Costa ${ }^{4}$
}

Fecha de recepción: 01 de Octubre de 2019 Fecha de aceptación: 05 de Febrero de 2020

Cómo citar: Narváez., D.A., Pérez., B.L., Giubergia., A.A., y Gil-Costa., G.V. (2020). Control y administración de datos en una mina subterránea de oro y plata. Tecnura, 24(64). 66-80

https://doi.org/10.14483/22487638.15587

\section{RESUMEN}

Objetivo: El objetivo de este trabajo es mostrar cómo el uso de un sistema de control de producción que registra y gestiona las operaciones mineras, enfocado en el seguimiento y control de los equipos, procesos y activos, mejora la eficiencia del sistema de información de una empresa, de modo que tenga un soporte adecuado.

Metodología: En este trabajo se utiliza el software Pitram versión 1.0, desarrollado por la empresa Micromine, que permite detectar las falencias en el sistema de información del área de producción en una pequeña mina subter- ránea de oro y plata, ubicada en Argentina. La metodología consistió en cinco pasos fundamentales: a) capacitación, b) recolección de datos, c) ingreso de datos al sistema, c) validación del modelo y d) análisis y resultados.

Resultados: Se obtuvo una mayor cantidad y variedad de datos con respecto a la información que registraba la empresa al momento de realizar el presente trabajo, que fueron propicios para tomar decisiones sobre el control de insumos, seguimiento de equipos primarios, seguimiento de operarios de equipos primarios, cálculo de KPI y control de objetivos. Además, se optimizó la administración de los datos, lo

Ingeniero en Minas, Departamento de Minería, Facultad de Ciencias Físico-Matemáticas y Naturales, Universidad Nacional de San Luis. Chacabuco 917 (5700), San Luis, Argentina Contacto: narvaez_41@hotmail.com

ORCID: https://orcid.org/0000-0002-4767-784X

2 Profesora en Ciencias de la Computación, auxiliar de Primera Categoría del Departamento de Minería, Facultad de Ciencias Físico-Matemáticas y Naturales, Universidad Nacional de San Luis. Chacabuco 917 (5700), San Luis, Argentina. Contacto: blperez@unsl.edu.ar ORCID: https://orcid.org/0000-0003-4505-9113

3 Magíster en Ingeniería del Software, ingeniera en Minería, profesor adjunto del Departamento de Minería, Facultad de Ciencias Físico-Matemáticas y Naturales, Universidad Nacional de San Luis. Chacabuco 917 (5700), San Luis, Argentina. Contacto: aagiuber@unsl.edu.ar ORCID: https://orcid.org/0000-0002-9780-6291

4 Doctora en Ciencias de la Computación, licenciada en Ciencias de la Computación. Profesor Asociado del Departamento de Minería, Facultad de Ciencias Físico-Matemáticas y Naturales, Universidad Nacional de San Luis. Chacabuco 917 (5700), San Luis, Argentina. Investigadora del CCT-SL (Conicet-CCT) San Luis. Argentina. Contacto: gvcosta@email.unsl.edu.ar

ORCID: https://orcid.org/0000-0003-4637-9725 
que significó la disponibilidad de estos en todo momento, menor cantidad horas hombres en el manejo de datos, menor consumo de recursos de almacenamiento, mayor seguridad, etc.

Conclusiones: El software implementado satisfizo la necesidad de información de la empresa, al minimizar la incertidumbre y reducir la posibilidad de cometer errores que podrían causar pérdidas económicas. Además, permitió mejorar la eficiencia operativa y optimizar la distribución de recursos, y así, una mejor rentabilidad.

Palabras clave: base de datos; planificación minera; software minero.

\section{ABSTRACT}

Objective: The objective of this work is to show how the use of a production control system that records and manages mining operations, focused on the monitoring and control of equipment, processes and assets, improves the efficiency of a company's information system, so that it has adequate support.

Methodology: This work uses Pitram software version 1.0, developed by the company Micromine, which allows detecting the flaws in the information system of the production area in a small underground gold and silver mine, located in Argentina. The methodology consisted of five fundamental steps: 1. Training, 2. Data collection, 3. Data entry to the system, 4 . Validation of the model, 5. Analysis and results.

Results: A greater amount and variety of data were obtained that were conducive to making decisions about input control, monitoring of primary equipment, monitoring of operators of primary equipment, calculation of $\mathrm{KPI}$ and control of objectives. In addition, data management was optimized, which meant their availability at all times, fewer man hours in data management, lower consumption of storage resources, greater security, etc.

Conclusions: The software implemented was able to meet the company's information need by minimizing uncertainty and reducing the possibility of making mistakes that could cause economic losses. In addition, it improved operational efficiency and optimized the distribution of resources, achieving better profitability.

Keywords: Database; Mining Planning; Mining Software.

\section{INTRODUCCIÓN}

La información puede ser utilizada como un recurso para el alcance del éxito en coordinación con los tradicionales recursos (materiales, económicos, humanos y financieros). Por tanto, su búsqueda y captura generan una ventaja competitiva para las empresas, porque es uno de los activos más importantes de estas, que además deberían ser protegidos adecuadamente mediante el uso de las tecnologías de la información y comunicación (TIC) (Ascanio, Trillos y Bautista, 2015).

\section{Planteamiento del problema, contexto y antecedentes}

El caso de estudio de este trabajo (figura 1) se desarrolla en una pequeña mina subterránea de oro y plata que se encuentra en la zona cordillerana de Argentina (Sistema de Información Geológica Ambiental Minera, 2019). Desde el inicio de la vida de la mina, en 2009, se contaba con escaso registro de datos, por lo que resultó imperativo solucionar este problema. Teniendo en cuenta que los tiempos favorables que se vivieron en la minería (gracias a los precios altos de los commodities) habían finalizado, surgió 
la necesidad de tener un control más estricto de recursos e insumos. Esto se logra teniendo un conocimiento integral de los procesos, por lo que es indispensable contar con un soporte de información que aclare cómo funciona cada variable de estos y tomar las decisiones con menor riesgo de cometer errores. En consecuencia, en 2015 se dio inicio al proyecto de implementación de un software específico Pitram (Micromine, 2016) que posee la capacidad de almacenar datos para luego re- cuperarlos a través de una gran variedad de informes muy útiles a la hora de analizar la eficiencia de la producción, controlar el consumo de insumos y la distribución de recursos. Por tanto, da a conocer con anticipación si los objetivos planificados se han de cumplir y, de no ser así, se tiene la posibilidad de tomar acciones correctivas.

El problema central que se busca resolver con la implementación del software

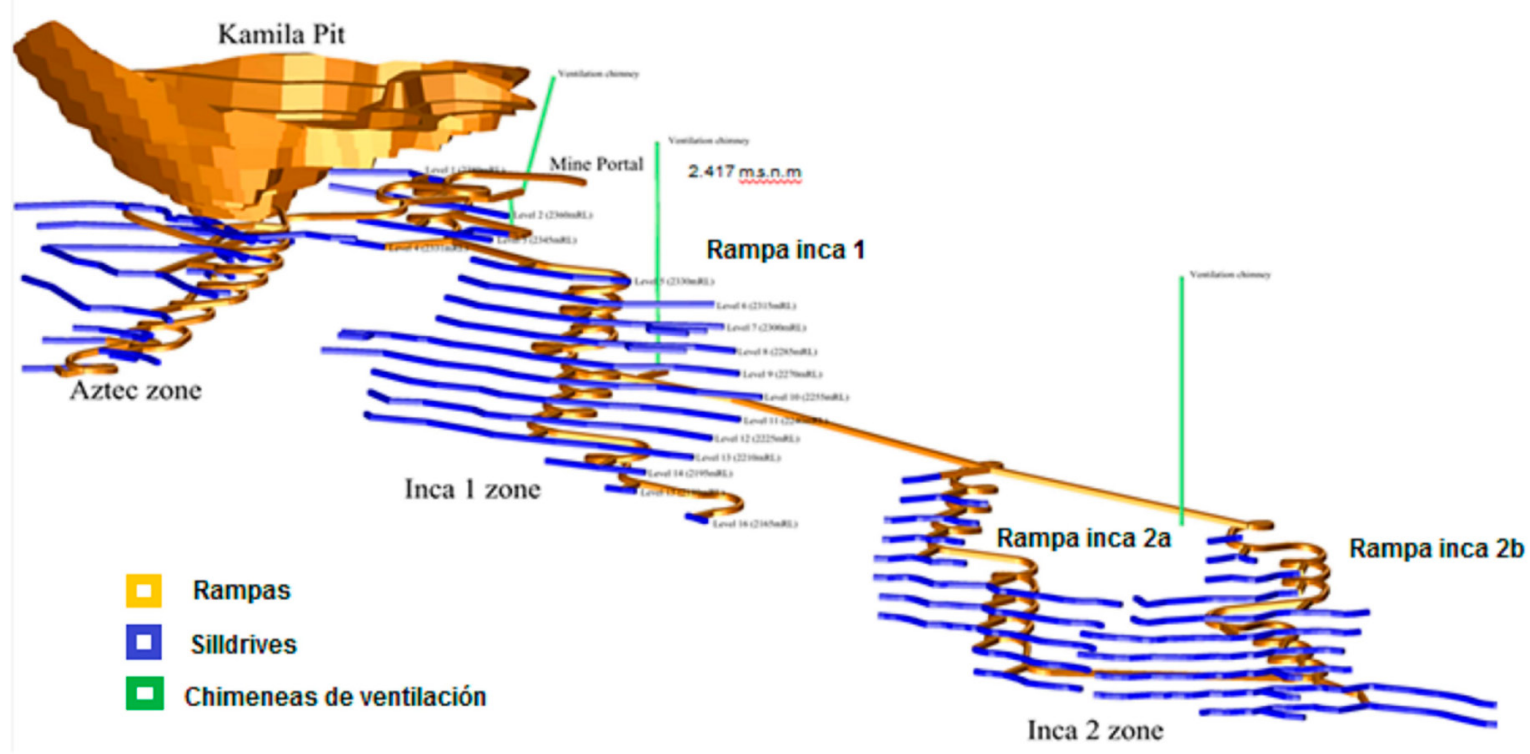

Figura 1. Vista general de la mina donde se muestran las tres zonas de explotación y las rampas de acceso. Espaciamiento entre niveles, en general, es de $15 \mathrm{~m}$ a $20 \mathrm{~m}$

Fuente: Mina Casposo. (Narváez, 2016)

es minimizar el escaso control de las operaciones de la mina, que causa incertidumbre acerca de algunos indicadores de producción importantes.

La disponibilidad de la información era otro problema que requería solución, ya que si un área necesitaba algún e informe perteneciente a otra área, debía solicitarlo vía correo electrónico, causando muchos contratiempos. Tener información disponible en cualquier momento, a la cual se pueda acceder desde cualquier computadora conectada a la red de la compañía, fue la razón por la que se decidió invertir en este proyecto. Durante varios años cada área o departamento de la compañía llevó el registro de datos en documentos (en su mayoría en planillas Excel) que se archivaban en carpetas a las que solo su personal tenía acceso (sistema antiguo).

En el transcurso de este trabajo se destacan los beneficios obtenidos por la utilización 
de este software, como: a) mayor disponibilidad de información, b) mejora en los tiempos de manejo y administración de datos, c) controles más estrictos de consumos de recursos y de trabajos realizados por empresas contratistas.

\section{Estado del arte}

En aras de crear y sostener una ventaja competitiva, según Hansen y Mowen (2006),

[...] la administración de la calidad total, el tiempo como elemento competitivo, los avances en la información y la tecnología de manufactura, la globalización de los mercados, el crecimiento del sector servicios, la liberación y una conciencia iluminada en relación con las prácticas éticas y ambientales de las empresas han impulsado cambios en el manejo de los datos. (p. v, prefacio)

El trabajo de Sánchez Martelo (2015) muestra la integración de dispositivos tecnológicos (una integración absoluta entre el dispositivo y el ser humano) en los sistemas de información. De esta manera hace notar que la persona se debe centrar solo en las tareas que debe realizar y no en las herramientas a utilizar. También resalta que cuanto más intuitivo es el uso de la tecnología, esta se considera más inteligente y que debe brindar calma (tranquilidad, confiabilidad y usabilidad) a los usuarios.

Contar con una base de datos referida a las actividades generales de la minería, como la explotación de minerales, operaciones unitarias, servicios auxiliares, producción mensual de minerales es fundamental para el buen control y aplicación de indicadores, así como para el control del desempeño de operaciones mineras (Escalante, 2011). El diseño y uso de indicadores de gestión en explotación y avances en la minería es a través de la elaboración de tablas de base de datos, cuadros de resumen y cuadros de control del desempeño de las operaciones para el logro de objetivos (Escalante, 2011).

Josep Tarruella (2009) hace hincapié en un artículo publicado por la revista Minería Chilena:

[...] la industria minera tiene una enorme complejidad en el manejo de sus datos, lo que provoca una necesidad de responder a normativas internacionales, integrar tecnologías utilizadas en distintas empresas o unidades, tener un inventario certero y un manejo confiable de la información del mineral, que permita evitar errores. Por ejemplo, en los sistemas de medición y contabilidad de producción, representa un desafío en el que se juega incluso la continuidad operativa, donde un dato erróneo o una demora en el procesamiento de la información puede generar pérdidas millonarias.

León Delgado (2019) determinó los índices técnicos de gestión que intervienen en la actividad minera subterránea mediante la recolección de datos, determinación de variables y parámetros de mayor relevancia que se deben medir. Estos ayudan a la gestión de procesos mineros para que la gerencia de la operación pueda actuar de forma efectiva y segura mejorando el desempeño de la industria minera.

En el trabajo de Cruz Choquepata (2016) se menciona que, al desarrollar proyectos operativos en minas a corto plazo, la implementación de herramientas informáticas proporciona mayor confiabilidad y control en el manejo de la información. Becerra-Fernández y Herrera-Ramírez (2018) elaboraron un modelo para planeación de 
capacidades operativas donde se buscó la mejor utilización de los recursos asignados a la prestación del servicio, según la rentabilidad de la unidad de negocio. Así se demostró que una mejor configuración de los recursos involucrados en dicha prestación de servicio le permite a las empresas obtener mejores índices de utilización y rentabilidad.

\section{Objetivos}

Implementar un sistema de administración de datos de producción más eficiente, con el cual se pueda satisfacer la necesidad de información, aumentando el conocimiento general de las operaciones de mina para la toma de decisiones dentro de la organización.

\section{Alcances}

El alcance de este trabajo es lograr, a través del uso del software, la generación de informes detallados que sean de interés para el área de producción, servicios técnicos y altas gerencias; además, registrar los movimientos de materiales con sus respectivas leyes, tareas realizadas por los equipos, consumibles, mediciones y avance en túneles de desarrollo según topografía.

\section{METODOLOGÍA}

- Para poner en marcha el proyecto se utiliza la siguiente metodología (figura 2):

- Realizar capacitaciones acerca del software, su funcionamiento y estructura.

- Realizar reuniones con los jefes de departamentos para establecer los datos de interés para cada uno de ellos.

- Trabajar en conjunto con personal de la empresa del software para suministrar la información que precisan para estructurarlo y personalizarlo, según las necesidades de la empresa minera.

- Extraer los datos de los registros existentes y recolectar otros datos a través de la creación de planillas diarias de trabajo para los equipos.

- Capacitar al personal en el uso de las planillas.

- Ingresar los datos a la base de datos del software.

- Rastrear errores y solucionarlos.

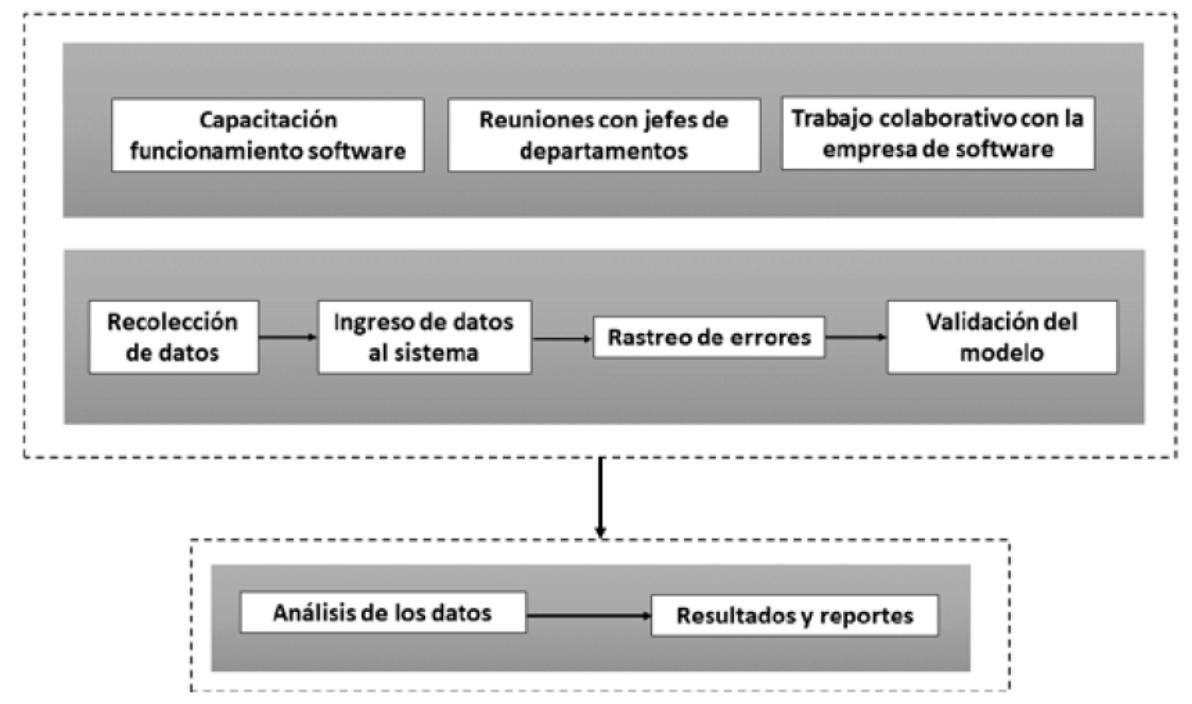

Figura 2. Esquema de la metodología empleada para generar el diseño de la base de datos Fuente: elaboración propia. 
El trabajo previo a la puesta en marcha del software abarca la etapa del diseño conceptual de la base de datos y la recolección de datos.

El software utiliza la tecnología de base de datos para controlar y gestionar la información, es por ello que el correcto funcionamiento de este depende de cómo fue diseñada la base de datos. Se puede decir que, en líneas generales, el diseño de un sistema de información, en lo conciernente a las bases de datos, tiene tres fases: modelo conceptual, modelo lógico, modelo físico (Camps Paré et al., 2016; Gómez Ballester et al., 2007). En la etapa de modelo conceptual se comienzan a realizar tareas para la implementación del software. Las etapas de modelo lógico y modelo físico son responsabilidad exclusiva del personal de la empresa del software, por lo que no se tienen en cuenta en este trabajo.

Por otra parte, la información que entrega el software está basada en los datos ingresados en este, por lo cual se debe tener un especial cuidado en la recolección, ingreso, validación y actualización de datos si se quiere obtener información confiable.

\section{Modelo conceptual de base de datos}

Según Gómez Ballester et al. (2007), el modelo conceptual "es la fase en la que se formalizan las estructuras que se observan en el mundo real produciendo lo que se denomina esquema conceptual". Por lo que en la etapa del diseño de la base de datos se representa la realidad a un alto nivel de abstracción, con modelos conceptuales de fácil comprensión.

Silberschatz, Korth y Sudarshan (2006) refieren que como parte inherente de un proceso de diseño conceptual "se deben tomar decisiones sobre qué atributos se desea capturar en la base de datos y cómo agruparlos para formar las diferentes tablas" (p. 12). Por tanto, el modelo de datos entidad-relación (E-R) basa la percepción del mundo real como una colección de objetos básicos, denominados entidades, y las relaciones entre estos objetos. Las entidades se describen mediante un conjunto de atributos (Silberschatz, Korth y Sudarshan, 2006).

Es importante definir tres conceptos básicos en el modelo E-R:

- Entidad. Es el objeto donde se almacena la información en la base de datos. Por ejemplo, un equipo determinado o un operario. De este modo se habla de la entidad personal, mientras que cada operario en concreto sería una ocurrencia (instancia) o un ejemplar de la entidad personal. La entidad se representa con un rectángulo (Sánchez, 2004) (figura 3).

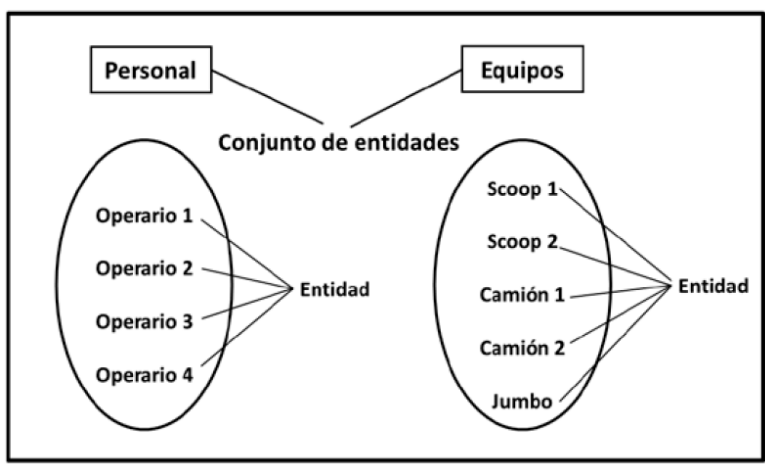

Figura 3. Ejemplo de entidad y conjunto de enti dades. Representación gráfica en modelo E-R

Fuente: elaboración propia.

- Relación. Elemento del modelo que permite relacionar los datos entre sí. Este se simboliza con un rombo (Sánchez, 2004). Por ejemplo, en caso de que se tenga una entidad personal y otra entidad equipos, ambas se relacio- 
nan ya que el personal opera equipos y los equipos son operados por el personal (figura 4).

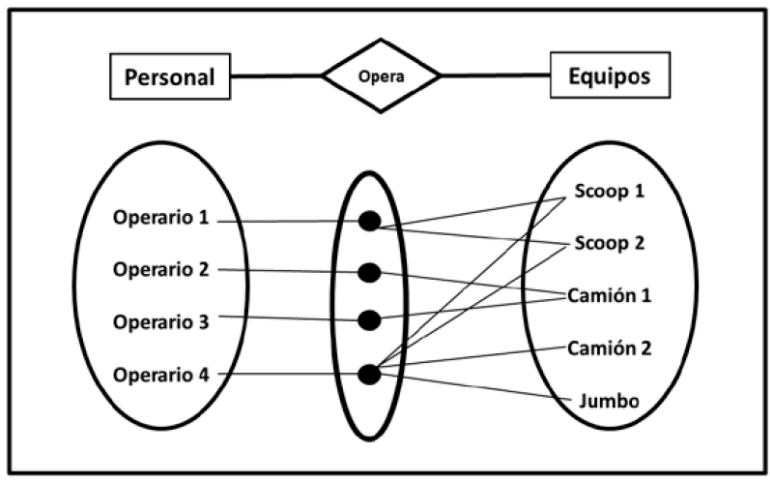

Figura 4. Relación entre entidades

Fuente: elaboración propia.

- Atributos. Describen propiedades de las entidades y las relaciones. Estos se representan con un círculo, donde se ubica el nombre del atributo (Sánchez, 2004). Por ejemplo, una entidad operario tiene atributos como el nombre, identificación y edad que lo describen (figura 5).

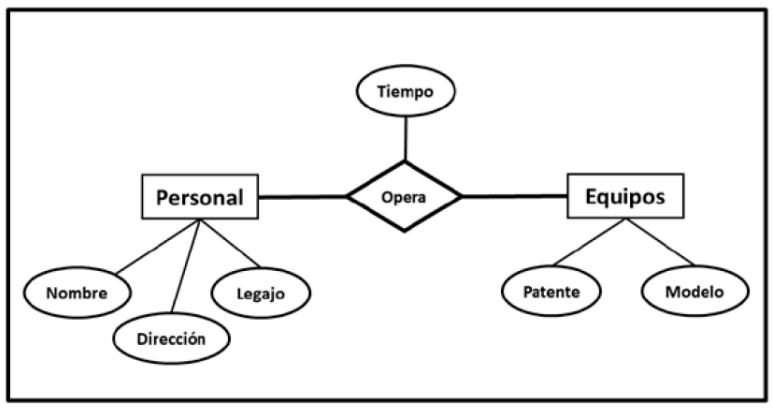

Figura 5. Relación de dos entidades con sus respectivos atributos

Fuente: elaboración propia.

Para ejemplificar las definiciones de entidad, relación y atributos, en este trabajo se usaron solamente dos entidades, una relación y seis atributos. En un modelo real existen muchos más componentes y varían según el caso, esto da la posibilidad de crear una gran cantidad de relaciones entre entidades. La ventaja de estas relaciones es que se pueden cruzar datos entre entidades, por ejemplo, se puede llegar a saber que un operario definido por un nombre, dirección y legajo, operó un equipo determinado por su modelo y patente.

\section{Información utilizada para la etapa del diseño conceptual}

En la figura 6. se muestra el índice del documento con la información requerida por la empresa de software para la etapa de diseño conceptual.

En la imagen se observan una serie de títulos (Equipment, Location, Measures, Personnel, Equipment States, Shift, Grade Control y Location States) que ordenan la información requerida. Este documento provee a los programadores los datos y la relación que existe entre ellos, de esta forma pueden realizar un modelo abstracto de la realidad que se necesita representar. Básicamente, para crear el esquema conceptual se requiere definir las entidades, cómo se relacionan entre ellas y sus respectivos atributos. Luego, se puede utilizar el modelo de datos E-R para crear el esquema conceptual de la base de datos.

Por ejemplo, en la tabla 1. se presentan los datos de los equipos de los cuales se desea obtener algún tipo de registro. Se describen las características como el modelo, la función y el código, lo que permitirá realizar reportes con mayor o menor detalle. A partir de estos reportes es posible consultar acerca de la cantidad de material transportado por cada uno de los camiones 
A40F. También se puede crear un reporte del material movido por los equipos que tiene la función "Equipos de acarreo", esto arrojará la suma de material transportado por cada los camión.

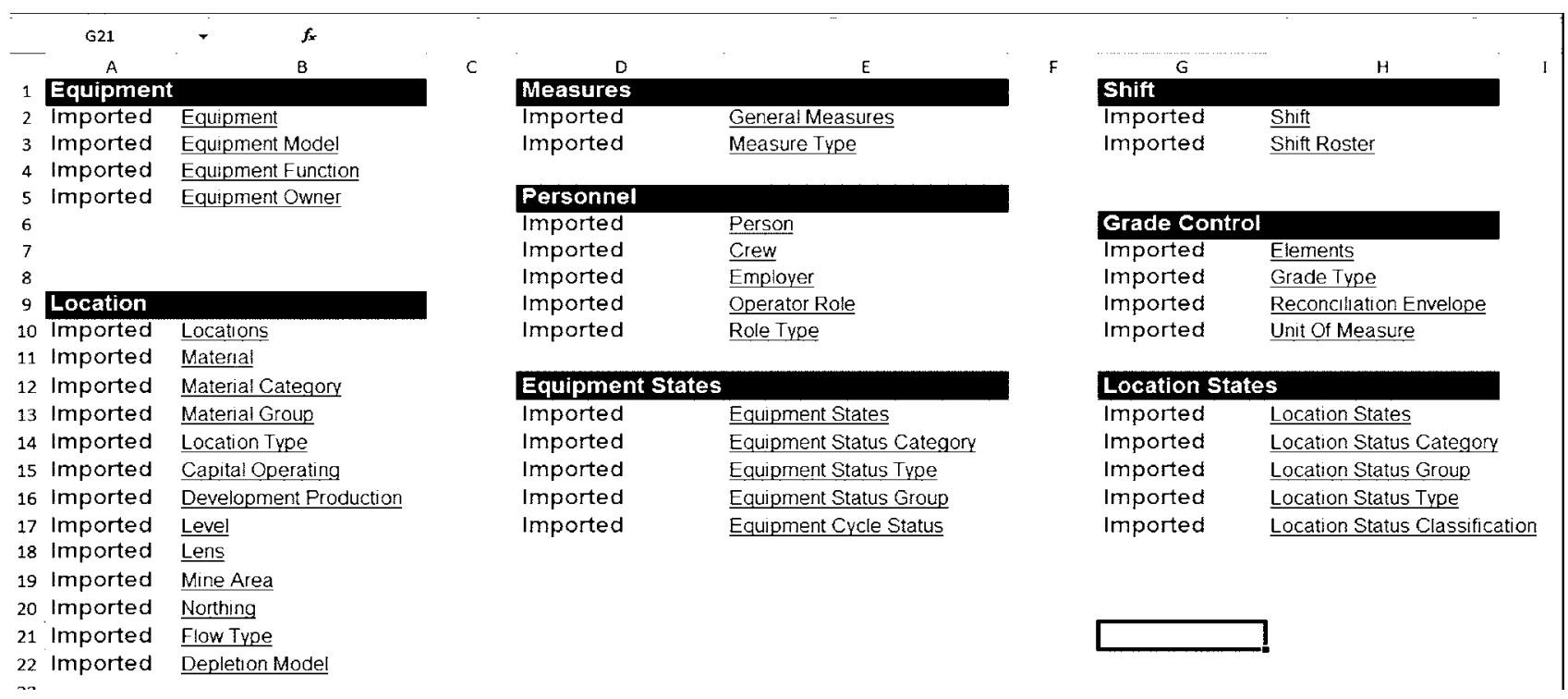

Figura 6. Índice del documento que registra la información necesaria para la etapa de diseño conceptual Fuente: elaboración propia.

\section{Manejo de datos}

Una vez que el software está configurado, se ingresan los datos, los cuales son almacenados en la base de datos para realizar consultas. La forma de manejarlos es muy importante, dado que es la base de la información que brinda el software en sus reportes. A la luz de esto, es necesario tener precauciones para lograr que los datos introducidos al software sean una representación fiel de la realidad. Para esto se adoptan tres pasos que garantizan un manejo óptimo de datos.

\section{Recolección}

Para poder satisfacer la necesidad de información de la empresa es preciso obtener los datos correctos. Se diseñó una serie de planillas diarias de trabajo que recolectaron la información necesaria, donde se tuvo en cuenta: los KPI (key performance indicator) (Gil, 2011), facilidad para entender, Ilenar y leer dichas planillas, la forma de trabajo del equipo y compatibilidad con el software.

Validación e ingreso de datos

Debido a que los datos que se ingresan en el software son la base de la información, es necesario llevar a cabo un estricto control de las planillas de trabajo diario para verificar que los datos contenidos son fieles a la realidad antes de ingresarlos a la base de datos. Esta tarea tiene un grado alto de dificultad, pero es indispensable para tener una base de datos confiable. Es responsabilidad del data entry verificar los datos antes de ser ingresados. 
Tabla 1. Detalle de la flota de equipos

\begin{tabular}{|c|c|c|c|c|c|c|}
\hline \multicolumn{7}{|c|}{ FLOTA DE EQUIPOS } \\
\hline Función & Equipo & Tipo & $\begin{array}{c}\text { Carga } \\
\text { nominal }\end{array}$ & $\begin{array}{c}\text { Carga } \\
\text { real }\end{array}$ & Fabricante & Código \\
\hline \multirow{3}{*}{$\begin{array}{c}\text { Equipo de } \\
\text { perforación y } \\
\text { desarrollo }\end{array}$} & Jumbo DD321 & primario & & & Sandvik & DB-01 \\
\hline & Jumbo DD321 & primario & & & Sandvik & DB-02 \\
\hline & Jumbo DD321 & primario & & & Sandvik & DB-03 \\
\hline \multirow{2}{*}{ Long Hole } & Solo DL321-7 & primario & & & Sandvik & PD-01 \\
\hline & Simba 1254 & primario & & & Atlas Copco & PD-02 \\
\hline \multirow{4}{*}{$\begin{array}{c}\text { Equipo de } \\
\text { extracción } \\
\text { LHD }\end{array}$} & Scoop R1600G & primario & $10 \mathrm{t}$ & $5,6 \mathrm{t}$ & Caterpillar & LHD-1 \\
\hline & Scoop R1700G & primario & $12,5 \mathrm{t}$ & $7,6 \mathrm{t}$ & Caterpillar & LHD-2 \\
\hline & Scoop R1600G & primario & $10,2 \mathrm{t}$ & $5,6 \mathrm{t}$ & Caterpillar & LHD-3 \\
\hline & Scoop R1300G & primario & $6,8 \mathrm{t}$ & $3,8 \mathrm{t}$ & Caterpillar & LHD-4 \\
\hline \multirow{4}{*}{$\begin{array}{l}\text { Equipos de } \\
\text { acarreo }\end{array}$} & $\begin{array}{c}\text { Camión Lagarto } \\
\text { A40F }\end{array}$ & primario & $39 t$ & $23 \mathrm{t}$ & Volvo & MT-01 \\
\hline & $\begin{array}{c}\text { Camión Lagarto } \\
\text { A40F }\end{array}$ & primario & $39 t$ & $23 \mathrm{t}$ & Volvo & MT-02 \\
\hline & $\begin{array}{c}\text { Camión Lagarto } \\
\mathrm{A} 40 \mathrm{~F}\end{array}$ & primario & $39 t$ & $23 \mathrm{t}$ & Volvo & MT-03 \\
\hline & $\begin{array}{c}\text { Camión Lagarto } \\
\text { A40F }\end{array}$ & primario & $39 t$ & $23 \mathrm{t}$ & Volvo & MT-04 \\
\hline \multirow{4}{*}{$\begin{array}{l}\text { Equipos de } \\
\text { servicio }\end{array}$} & $\begin{array}{c}\text { Front End } \\
\text { Loader L90F }\end{array}$ & secundario & & & Volvo & L90-01 \\
\hline & $\begin{array}{l}\text { Scissor Lift } \\
\text { SLX5000DF }\end{array}$ & secundario & & & Walden & SL-01 \\
\hline & $\begin{array}{l}\text { Telehandler } \\
1740 \text { Haulotte }\end{array}$ & secundario & & & Faresin & TH-01 \\
\hline & $\begin{array}{l}\text { Anfo Loader } \\
\text { AFX5000MK }\end{array}$ & secundario & & & Walden & AL-01 \\
\hline \multirow{2}{*}{$\begin{array}{c}\text { Equipos } \\
\text { auxiliares }\end{array}$} & Alpha 20 & secundario & & & Semmco & BTZ-1 \\
\hline & Scoop ST1030 & secundario & & & Atlas Copco & BTZ-2 \\
\hline \multirow{3}{*}{$\begin{array}{c}\text { Equipos } \\
\text { auxiliares }\end{array}$} & Scoop ST730 & secundario & & & Atlas Copco & BTZ-3 \\
\hline & Manitou MT732 & secundario & & & Manitou & BTZ-4 \\
\hline & $\begin{array}{l}\text { Manitou } \\
\text { MT1030 }\end{array}$ & secundario & & & Manitou & BTZ-5 \\
\hline
\end{tabular}

\section{Actualización}

Para lograr que un sistema de información sea eficiente, una de las condiciones es que los datos se encuentren disponibles para ser consultados en tiempo y forma, es decir que deben ser actualizados en un periodo razonable, en este caso, día tras día.

Esta responsabilidad también recae sobre el data entry, quien es el encargado de que los datos de las planillas diarias de trabajo sean validados e introducidos a la base de datos dentro del periodo estipulado.

Mantener la aplicabilidad del software también se enmarca en la actualización. Aplicabilidad quiere decir que sea adaptable a la realidad del proceso del cual se quieren obtener datos. Esta tarea es responsabilidad del administrador del software de la empresa, el cual tiene acceso al servicio editor de referencias, para 
crear, editar y administrar los datos de referencia utilizados por el software. Esto incluye, entre otras tareas, la definición de equipos, ubicaciones y personal. La configuración de la base de datos es un proceso constante.

\section{RESULTADOS}

Los resultados y beneficios derivados de la implementación del software tienen un impacto positivo ante la necesidad de remediar o compensar la ausencia de información que se requiere al momento de tomar decisiones dentro de la empresa. Los beneficios de mayor impacto son:

\section{Control de insumos}

Existe una gran cantidad de reportes que ayudan al control de insumos en la mina. Por ejemplo, el control de insumos de fortificación en una mina es importante si se desean bajar los costos de producción. Uno de los consumibles de alto costo en la mina de este estudio son los de fortificación. En este caso, con la ayuda de los reportes emitidos por el software, se conocen la cantidad y tipo de split set utilizados, pero no se pudo realizar el análisis acerca de la eficiencia de su uso. Este es uno de los beneficios que brinda el software en la optimización de recursos y así reducir los costos.

\section{Seguimiento de equipos primarios}

En este punto, el sistema de información permite hacer el seguimiento de los equipos que tienen gran influencia en el proceso extractivo. Por ejemplo, para el seguimiento de los Jumbos es posible identificar si alguno de ellos no respeta los estándares de fortificación de Geomecánica. Adicionalmente, se obtiene un informe detallado con la cantidad de pernos colo- cados por Jumbo en cada frente, lo que posibilita compararlos con los cálculos de Geomecánica y efectuar un control para verificar el cumplimiento de los estándares de fortificación. Luego del análisis se puede identificar a los operarios de los equipos que no cumplieron con el estándar de fortificación y tomar medidas para remediar la situación, como hacer capacitaciones acerca de los estándares que rigen en la mina.

A través de la herramienta de seguimiento que brinda el software se puede determinar la eficiencia de la utilización de los equipos con la ayuda de los reportes gráficos que aquel entrega. La figura 7. es un ejemplo de un gráfico de torta, en el que se encuentran todas las tareas realizadas por el camión MT-01 con su respectiva distribución de tiempos expresados en porcentajes, para una fecha determinada.

Estos reportes ayudan a visualizar la forma en la cual los equipos están siendo utilizados e identificar aquellas tareas no operativas que ocupan un gran porcentaje de tiempo, para luego, si fuera necesario, llevar a cabo alguna acción correctiva. Seguimiento de operarios de equipos primarios.

A través de los reportes que se obtienen del programa, se adelantan los seguimientos a los operarios de equipos con mayor influencia en el proceso productivo. Esto permite darles a conocer los puntos en los que tienen que mejorar y de esta forma incentivarlos en su trabajo.

\section{Cálculo de KPI}

De los reportes proporcionados por el software referidos a $\mathrm{KPI}$, solo se tienen en cuenta los solicitados por la gerencia del área de producción: 
1. Perforación efectiva de desarrollo. Se puede validar la cantidad de metros perforados planificados con la perforación de los metros efectivos.

2. Velocidad de perforación de desarrollo. Permite conocer el tiempo que demora en perforar un frente en función de la velocidad de perforación que tiene un Jumbo.

3. Toneladas por hora de Scoop. Se obtiene un informe con los tonelajes movidos por los Scoop y los camiones, así como también los tiempos operativos invertidos en el movimiento de material. Con esta disponibilidad de datos se pueden calcular las toneladas por hora de Scoop.

4. Toneladas por hora de camión. Se obtiene el reporte de la cantidad de toneladas transportadas por los camiones y el tiempo utilizado.

5. Velocidad de perforación de long hole.

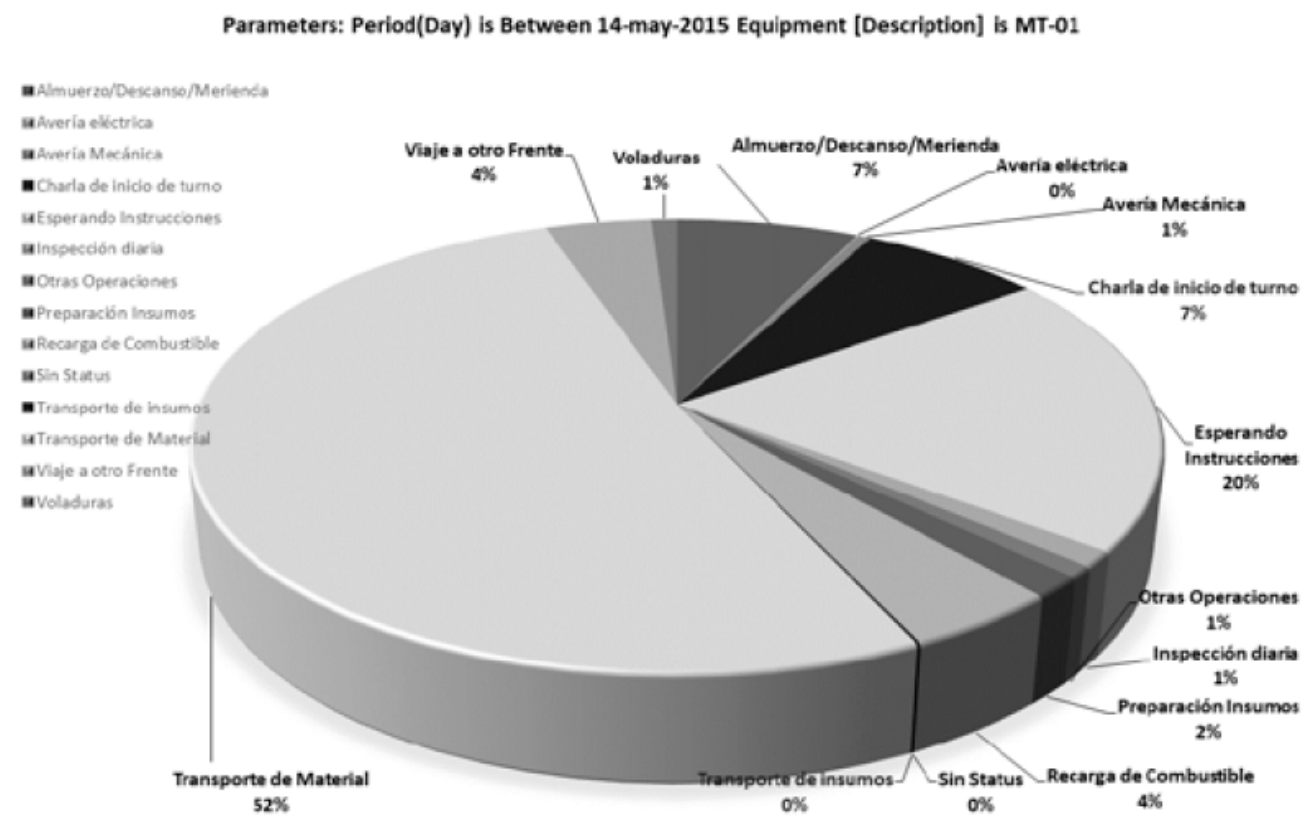

Figura 7. Reporte gráfico con los porcentajes de tiempos por cada tarea para el camión MT-01

Fuente: elaboración propia.

Se obtiene un reporte de los tiempos de las tareas operativas, con los cuales se puede calcular la velocidad de perforación long hole.

\section{Control de objetivos}

El software emite reportes de seguimiento de los objetivos para así analizar su cumplimiento. Esto es de gran utilidad, ya que, si se estima que alguno de los obje- tivos no se va a cumplir, pueden tomarse acciones correctivas con anterioridad para revertir la situación.

La figura 8. muestra un informe gráfico y numérico del objetivo diario y acumulado de toneladas de producción. Las barras en la imagen representan la producción en toneladas del día, mientras que la línea punteada determina el objetivo diario, es decir que todas las barras que sobrepasan esta línea cumplen la meta di- 
aria. La línea continua del medio es el objetivo diario acumulado, el cual debe ser comparado con la línea continua inferior para determinar si se cumple.

\section{Beneficios}

Los beneficios obtenidos representan, en primer lugar, la mayor cantidad de información, es decir que con este sistema de información, existe más variedad de datos propicios para tomar decisiones a partir de estos. En segundo lugar, la administración de los datos significó la disponibilidad de estos en todo momento, menor cantidad horas/hombres en el manejo de datos, menor consumo de recursos de almacenamiento, mayor seguridad, etc.
La tabla 2. muestra la comparación entre el antiguo sistema (registro de datos en planillas Excel, en general) y el nuevo sistema. Se puede apreciar en cada una de las comparaciones las ventajas del nuevo sistema implementado.

\section{CONCLUSIONES}

Una necesidad primaria de las organizaciones es una fuente de información confiable sobre la cual fundamentar sus decisiones. Antes de la implementación del software, el control y administración de datos eran ineficientes, y no registraban toda la información relevante para el área de Servicios Técnicos y Producción.

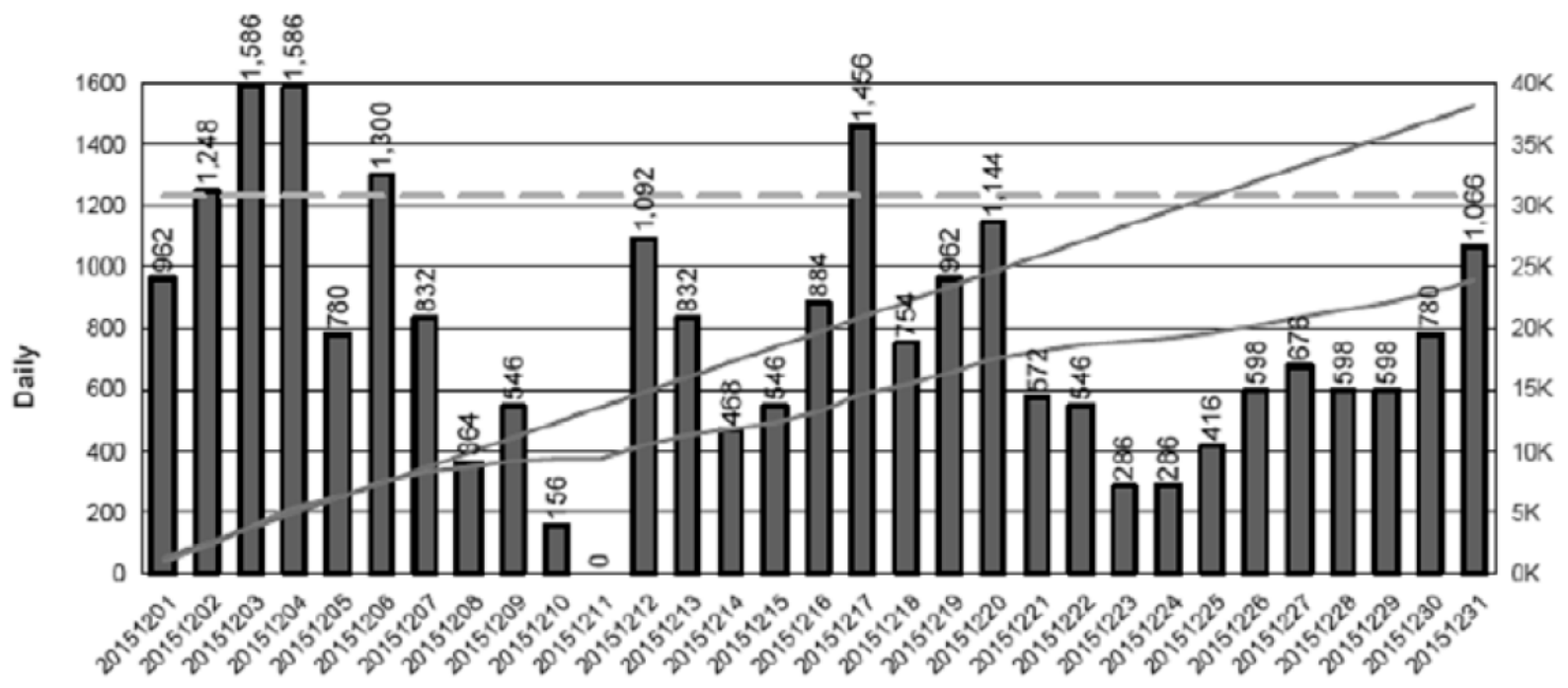

Figura 8. Reporte donde se muestra el objetivo diario y acumulado de toneladas de producción Fuente: elaboración propia. 
Las características y funciones de este software implementado satisfizo la necesidad de información de la empresa, minimizó la incertidumbre y redujo la posibilidad de cometer errores que podrían causar pérdidas económicas. También permitió mejorar la eficiencia operativa y optimizar la distribución de recursos, para así lograr mejor rentabilidad.

\section{REFERENCIAS}

Ascanio, J.G.A., Trillos, R.A.B. y Bautista, D.W.R. (2015). Implantación de un sistema de gestión de seguridad de información bajo la ISO 27001: Análisis del riesgo de la información. Tecnura, 19(46), 123-134. DOI: https://doi.org/10.14483/ udistrital.jour.tecnura.2015.4.a10

Tabla 2. Cuadro comparativo entre el sistema nuevo y antiguo.

\section{Comparación entre sistemas de información}

\section{Sistema nuevo}

\section{Sistema antiguo}

Mayor captura de datos.

Disponibilidad de información y de fácil acceso debido a la base de datos centralizada.

Datos no redundantes, demanda menos espacio de almacenamiento.
Datos insuficientes.

Información no disponible en todo momento debido a la existencia de carpetas con uso restringido.

Datos redundantes, provoca una gran demanda de espacio en los medios de almacenamiento.
Reducción en las horas hombres empleadas para el manejo de información. Pocas personas realizan el ingreso de datos.

Debido a que se trata de una base de datos centralizada, se facilita su actualización.

Consulta de información de manera fácil e intuitiva.

Alto nivel de seguridad informática.
Muchas personas ingresando datos, lo que causa que varias veces se ingrese la misma información. Esto provoca redundancia de datos.

Al ser un sistema de archivos es difícil realizar una actualización en algún dato y que el cambio sea hecho en todos los archivos.

Para realizar una consulta se debe conocer la ubicación del archivo que contiene la información que se busca.
Los archivos pueden ser borrados con facilidad, causando pérdida de información.

Fuente: elaboración propia. 
Becerra Fernández, M. y Herrera Ramírez, M.M. (2018). Planeación de capacidades operativas asociadas a la rentabilidad en empresas prestadoras de servicios. Un enfoque de dinámica de sistemas. Operational planning capacities associated with profitability of service companies. A system dynamics approach.. Tecnura, vol. 22, no. 57, (32-43). DOI: https:// doi.org/10.14483/22487638.9169 Recuperado de https://revistas.udistrital.edu.co/index.php/Tecnura/article/view/9169 https://doi. org/10.14483/22487638.9169

Camps Paré, R., Casillas Santillán, L.A., Costal Costa, D., Ginestá, M.G., Martín Escofet, C. y Pérez Mora, O. (2016). Bases de datos. Recuperado de https://librosysolucionarios.net/ bases-datos-rafael-camps-pare-luisalberto-casillas-santillan-dolors-costal-costa-marc-gilbert-ginesta-carmenmartin-escofet-oscar-perez-mora/

Cruz Choquepata, J.E. (2016). Planeamiento de minado a corto plazo con la implementación de herramientas informáticas en CIA Minera Catalina Huanca S.A.C. Trafigura Beheer B.V. Mining. [Tesis de pregrado]. Universidad Nacional de San Agustín. Arequipa, Perú. Recuperado de http://repositorio.unsa.edu.pe/handle/UNSA/3249

Escalante Sánchez, R. (2011). Aplicación de indicadores de gestión en operaciones en la Mina Catalina Huanca SAC. [Tesis de pregrado]. Escuela de Formación Profesional de Ingeniería de Minas. Ayacucho, Perú. Recuperado de http://repositorio.unsch.edu.pe/ bitstream/UNSCH/2143/1/TESIS\%20 M650_Esc.pdf
Gil Agudelo, D.V. (2011). Indicadores claves de rendimiento (KPI) Cummins de los Andes S. A. [Tesis de pregrado]. Corporación Universitaria Lasallista, Facultad de Ingeniarías, Ingeniería Industrial. Caldas, Antioquía. Recuperado de http://repository.lasallista.edu. co/dspace/bitstream/10567/720/1/Indicadores_claves_rendimiento_CummisdelosAndes.pdf 47.

Gómez Ballester, E., Suárez Cueto, A., Saquete Boro, E., Matínez Barco, P., Moreda Pozo, P. y Montoyo Guijarro, A. (2007). Apuntes de Bases de Datos 1. Recuperado de https://www.academia. edu/17508862/Apuntes_de_Bases_de_ Datos_1

Hansen, D.R. y Mowen, M.M. (2006). Cost management: Accounting and control. 5a. ed. Mason, Ohio: Thomson/ South-Western.

León Delgado, R.J. (2019). Determinación de índices técnicos de gestión en el desarrollo de labores mineras subterráneas en la mina San Salvador. [Tesis de pregrado]. Universidad del Azuay. Cuenca, Ecuador. Recuperado de http://dspace. uazuay.edu.ec/handle/datos/8927

Micromine (3 de enero de 2016). Pitram Mining Software. Recuperado de https:// www.micromine.com/pitram-mining-software/

Narváez, D.A. (2016). Control y Administración de Datos en la Mina Casposo con Pitram. [Tesis de pregrado]. Universidad Nacional de San Luis. San Luis, Argentina.

Sánchez, J. (2004). Diseño conceptual de bases de datos. Recuperado de https:// www.freelibros.me/base-de-datos/diseno-conceptual-de-bases-de-datos 
Sánchez Martelo, C.A. (2015). La computación ubicua: omnipresencia en los sistemas de información. Tecnura, 19, 121-128. DOI: https://doi. org/10.14483/22487638.10378

Silberschatz, A., Korth, H.F. y Sudarshan, S. (2006). Fundamentos de bases de datos. 5a. ed. McGraw-Hill.

Sistema de Información Geológica Ambiental Minera (2019). Cartas Minerometalogenética $250 \mathrm{~K}$. Recuperado de https://sigam.segemar.gov.ar/visor/

Tarruella, J. (2009). El manejo de datos en el sector minero. Minería Chilena. Recuperado de website: https://www.mch. cl/reportajes/el-manejo-de-datos-en-elsector-minero/ 